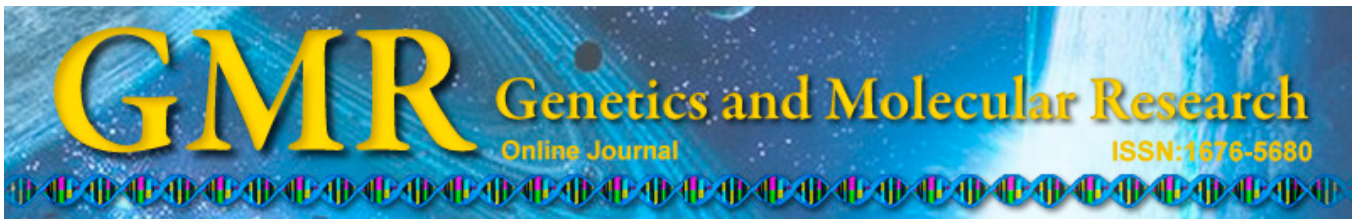

\title{
Computational identification and characterization of conserved miRNAs and their target genes in beet (Beta vulgaris)
}

\author{
J.L. Li, J. Cui and D.Y. Cheng \\ Food Science and Engineering, Harbin Institute of Technology, Harbin, China \\ Corresponding author: J. Cui \\ E-mail: cuijie@hit.edu.cn
}

Genet. Mol. Res. 14 (3): 9103-9108 (2015)

Received November 30, 2014

Accepted March 5, 2015

Published August 7, 2015

DOI http://dx.doi.org/10.4238/2015.August.7.19

\begin{abstract}
Highly conserved endogenous non-coding microRNAs (miRNAs) play important roles in plants and animals by silencing genes via destruction or blocking of translation of homologous mRNA. Sugar beet, Beta vulgaris, is one of the most important sugar crops in China, with properties that include wide adaptability and strong tolerance to salinity and impoverished soils. Seedlings of $B$. vulgaris can grow in soils containing up to $0.6 \%$ salt; it is important to understand the molecular mechanisms of salt tolerance to enrich genetic resources for breeding salt-tolerant sugar beets. Here, we report 13 mature miRNAs from 12 families, predicted using an in silico approach from 29,857 expressed sequence tags and 279,223 genome survey sequences. The psRNATarget server predicted 25 target genes for the 13 miRNAs. Most of the target genes appeared to encode transcription factors or were involved in metabolism, signal transduction, stress response, growth, and development. These results improve our understanding of the molecular mechanisms of miRNA in beet and may aid in the development of novel and precise techniques
\end{abstract}


for understanding post-transcriptional gene-silencing mechanisms in response to stress tolerance.

Key words: Beta vulgaris; Bioinformatics; EST; GSS; microRNA

\section{INTRODUCTION}

MicroRNAs (miRNAs) are small (19-23 nucleotides long) non-coding RNAs. Despite their small size, miRNAs act as key factors in regulating gene expression at the post-transcriptional level, either by endonucleolytic cleavage of mRNAs predominantly via RNA-induced silencing or by repressing protein translation (Llave et al., 2002; Dalmay, 2006; Jones-Rhoades et al., 2006; Sunkar et al., 2007). Increasing evidence suggests that miRNAs play important roles in many physiological and biochemical events, such as plant growth and response to environmental stress (Bartel, 2004; Wakeel et al., 2011; Khraiwesh et al., 2012). The identification and characterization of target miRNA genes in plants has been widely studied in recent years (Jones-Rhoades et al., 2004; Zhang et al., 2006a; Unver and Budak, 2009). Several high-throughput techniques, such as direct cloning and deep sequencing, have been used for experimental determination of miRNAs, along with homology-based comparative genomic analysis and genome survey sequences (GSS) from expressed sequence tags (ESTs) (Song et al., 2010; Kulcheski et al., 2011; Li et al., 2011; Barrera, 2012). According to the latest release of the miRNA database miRBase (www. mirbase.org), 28,645 miRNAs from different species have been identified and deposited. Sugar beet (Beta vulgaris ssp. vulgaris, Caryophyllales, Amaranthaceae) is an important temperate crop that provides approximately $30 \%$ of the world's annual sugar production and is a source of bioethanol and animal feed. The availability of a large number of ESTs of $B$. vulgaris provides an excellent source of experimental material for clarification of gene expression and regulation. Although miRNAs have been studied extensively in the past decade (Gandikota et al., 2007; Shukla et al., 2008; Ding et al., 2009; Sakaguchi and Watanabe, 2012), systematic studies of miRNAs for B. vulgaris are limited.

\section{MATERIAL AND METHODS}

To study potential miRNA precursor sequences, all 29,857 B. vulgaris ESTs and 279,223 B. vulgaris GSSs were downloaded from the National Center for Biotechnology Information (NCBI) GenBank EST and GSS databases. A total of 1328 unique (non-redundant) mature miRNAs from the publicly available miRBase database (Release 20; www.mirbase. org) were used as references for seeking conserved sequences in beet. The alignment tool (Altschul et al., 1990) BLAST version 2.2.27 (http://ftp.ncbi.nih.gov/blast/executables/blast + ) was used to predict conserved miRNAs in beet ESTs and GSSs. We used the online RNA Folding Form (http://mfold.rna.albany.edu/) to show secondary structures of pre-miRNAs. The plant small RNA (psRNA) Target server (http://plantgrn.noble.org/psRNATarget/) was used to search for target genes of putative miRNAs in B. vulgaris. The InterPro tool (http://www.ebi. ac.uk/interpro/) was then used to further annotate miRNA targets in beet to deduce the three important components, namely biological process, cellular component and molecular function, interrelated with each GO term. 


\section{RESULTS AND DISCUSSION}

\section{Identification of $B$. vulgaris miRNAs by EST and GSS analysis}

Most mature miRNAs are comparatively conserved among plant species (Dezulian et al., 2005, 2006), which has greatly helped in their identification using EST and GSS sequences. Conserved miRNAs in plants including Arabidopsis, maize, rice, soybean, and sugarcane can be identified by comparative genome-based analysis. We used the same comparative genomic approach to seek conserved miRNAs in beet ESTs and GSSs, because no miRNAs have been contributed to the miRBase database for $B$. vulgaris to date.

BLAST with beet ESTs and GSSs led to the discovery of 127 candidate miRNAs, of which 20 coding sequences were eliminated by BLASTX with the beet NR protein database. The secondary structure of the remaining precursor sequences of potential miRNA homologs was estimated using the Zuker folding algorithm, and the criteria as described earlier by Zhang et al. (2005) were applied to select potential miRNAs. Finally, we acquired 23 miRNAs from 14 families after taking MFE and MFEI into account.

To differentiate miRNAs from other small RNAs, various features of the conserved miRNAs in beet were further analyzed. All the predicted miRNAs were 19 or $22 \mathrm{nt}$ in length (Table 1) and occupied 30 and $70 \%$ of the total population, respectively, which is in agreement with previous reports (Zhang et al., 2006a, 2008). In contrast, the length of potential precursor beet miRNAs changed dramatically between 90 and $270 \mathrm{nt}$. This result was similar to findings in Arabidopsis thaliana, Zea mays, and Oryza sativa. The A + U content of the candidate miRNAs was determined and ranged from 41.1 to $69.1 \%$ (average $=57.37 \%$ ), which was in strong agreement with the findings of Zhang et al. (2006a). The proportions of A, U, G, and C nucleotides in precursor miRNA in beets were not equal; $\mathrm{U}$ and $\mathrm{A}$ were more abundant than $\mathrm{G}$ and $\mathrm{C}$.

The negative MFE and MFEI values of the candidate miRNA precursors were also calculated. MFE is an important parameter that reflects stability of perfect or near-perfect secondary hairpin structures of pre-miRNAs. A negative relationship between MFE and the thermodynamic stability of the secondary structure of the corresponding precursor sequence has been shown (Prabu and Mandal, 2010). The MFEI of precursor miRNAs varied significantly, from 0.74 to 1.21 (average $=0.94 \pm 0.02$ ), which was relatively higher than that of tRNAs (0.64), rRNAs (0.59), and mRNAs (0.62-0.66). This suggested that these recently evaluated B. vulgaris miRNAs are likely to be actual miRNAs rather than a non-coding RNA. The location of the mature miRNA within the $5^{\prime}$ or $3^{\prime}$ end of the stem-loop hairpin structure of potential miRNAs was also identified; five mature miRNAs were located on the $3^{\prime}$ end and the remaining eight were located on the $5^{\prime}$ end (Figure S1). Furthermore, a conserved miRNA search showed that either the precursor miRNA of beet (Bvu-miR3) or its nearest homolog (osa-miR169h) had the same stem-loop hairpin structure, in which the precursor miRNAs was approximately $120 \mathrm{nt}$ and the mature miRNA was located at the $5^{\prime}$ end (Jones-Rhoades et al., 2004).

Earlier experiments showed that either pre-miRNAs or mature miRNAs of plants are conserved among species (Zhang et al., 2006b). Our multiple sequence alignment and maximum-likelihood analyses indicated that Bvu-miR1 of $B$. vulgaris is closely associated with members of the miR160 family, and that Bvu-miR2 shares a high degree of sequence homology with the miR168 family. In addition, Bvu-miR3 and Bvu-miR4 are part of the miR169 family. In contrast to these results, the remaining miRNAs from Bvu-miR5 to Bvu-miR13 
had high sequence homology with miR396, miR397, miR403, miR414, miR530, miR2919, miR5021, miR5368, and miR5658, respectively. The phylogenetic tree illustrated that the evolutionary relationships in beet miRNAs were not the same as those in other species (Figure S2). In addition, the phylogenetic analysis leads to the conclusion that miRNAs evolve at different rates within and between plants.

Table 1. Characterization of novel identified Beta vulgaris miRNAs.

\begin{tabular}{|c|c|c|c|c|c|c|c|c|c|c|c|}
\hline Beet miRNAs & Mature miRNAs & source & $\begin{array}{l}\text { Homologous } \\
\text { miRNA }\end{array}$ & Strand & Loc & LP & LM & NM & $\begin{array}{c}\mathrm{A}+\mathrm{U} \\
(\%)\end{array}$ & $\begin{array}{l}\text { MFE } \\
(\triangle \mathrm{G})\end{array}$ & MFEI \\
\hline 21 & GGCUCCCUC & $\mathrm{gb} \mid \mathrm{DX} 10$ & osa-m & $+/-$ & $3^{\prime}$ & 90 & 21 & 0 & 43.3 & -61.6 & 1.21 \\
\hline & UCG & $\mathrm{gb} \mid \mathrm{D}$ & gma-r & $+/-$ & $5^{\prime}$ & 200 & 21 & 0 & & -62.3 & 0.79 \\
\hline vu-miR3 & UAGCCAAGGA & $\mathrm{gb} \mid \mathrm{JY}$ & osa-miR169h & $+/-$ & $5^{\prime}$ & 120 & 21 & 0 & & -40.2 & 0.98 \\
\hline $\mathrm{vy-miR4}$ & GACUUGCC & $\mathrm{gb} \mid \mathrm{HN}$ & gma-miR169v & $+/-$ & $5^{\prime}$ & 200 & 19 & 0 & & & 0.85 \\
\hline miD 5 & $\mathrm{UUCC}$ & $\mathrm{gb} \mid \mathrm{B}$ & miR 396 & +1 & $5^{\prime}$ & 155 & s & 0 & & & 1.04 \\
\hline $\mathrm{vu}$ & UCA & $\mathrm{gb} / \mathrm{JY}$ & osa-miR39 & + & $3^{\prime}$ & 90 & 2 & 0 & 9 & & 1.3 \\
\hline Bvu & UUA & & ath-miR4 & & $\theta$ & & & 0 & & & 0.91 \\
\hline & & & & & $J$ & & 2 & 1 & & & 0.98 \\
\hline the & & & 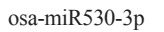 & + & $3^{\prime}$ & 0 & 21 & 3 & 2 & -2 & 0.71 \\
\hline Bvu & A & 8 & osa-1 & +1 & $3^{\prime}$ & 217 & 19 & 2 & 41.1 & -129.14 & 1.08 \\
\hline Bvu-miR11 & GAGAAGAAGAAGAAGAAAA & $\mathrm{gb} \mid \mathrm{JY}$ & ath-miR5021 & $+/-$ & $3^{\prime}$ & 109 & 19 & 1 & 52.3 & -41.7 & 0.81 \\
\hline Bvu-miR12 & GGACAGUCUCAGGUAGACA & $\mathrm{gb} \mid \mathrm{DX} 1$ & gma-miR5368 & $+/-$ & $5^{\prime}$ & 180 & 19 & 0 & 45.0 & -72.8 & 0.79 \\
\hline Bvu-miR13 & AUGAUGAUGAUGAUGAUGAAA & $\mathrm{gb|JY} 330502.1 \mid$ & ath-miR5658 & $+/-$ & $5^{\prime}$ & 270 & 21 & 0 & 60.3 & -78.8 & 0.74 \\
\hline
\end{tabular}

Loc $=$ Location of miRNA, $\mathrm{LP}=$ length of precursor miRNA, $\mathrm{LM}=$ length of mature miRNA, NM = number of mismatch between predicted and homologous miRNA, MFE $=$ minimal folding free energy, AMFE $=$ adjusted minimal folding free energy, MFEI = minimal folding free energy index.

\section{Target prediction}

Earlier studies have shown that plant miRNAs predominantly target transcription factors (Llave et al., 2002; Chen, 2004), but subsequent studies have revealed that miRNAs also target plant functional genes that are involved in various physiological processes, including root growth and development, stress responses, signal transduction, leaf morphogenesis, plant defenses, and biogenesis of sRNA (Khraiwesh et al., 2012). Unlike in animals, miRNAs in plants identify their target mRNAs through perfect or near-perfect complementarity and initiate cleavage. On the basis of the mechanism of miRNAs in plants, a homology search-based method was used for miRNA target prediction in B. vulgaris using psRNATarget. The newly identified beet miRNAs were used as queries to predict potential mRNA targets in the DFCI Gene Index (BVGI). This search revealed that 8 of the 13 miRNAs identified 25 target genes, with each miRNA predicting more than one gene (Table S1). No target could be predicted from the current database of beet for Bvu-miR4, Bvu-miR9, Bvu-miR10, Bvu-miR11, or Bvu-miR12, and no GO term annotation was found for Bvu-miR5. Most of the newly identified B. vulgaris miRNAs targeted important genes/proteins involved in metabolism, stress response, transcriptional regulation, signal transduction, growth, and development (Table S1). The newly identified miRNAs also target a number of transcription factors (Table S1). Either diphenol oxidase or the PHYA4 photoreceptor is targeted by Bvu-miR6. The protein targeted by Bvu-miR7 is involved in various oxidation-reduction processes (Lin et al., 2009). Proteins targeted by Bvu-miR8 are involved in a wide range of molecular processes (Yanhui et al., 2006; Zhang et al., 2013); similarly, BvumiR13 has a wide range of predicted targets (Table S1). In summary, the miRNA family miR414 and miR5658 showed the maximum numbers of target genes, followed by miR168 and miR397; the remaining miRNA families each had one target. 


\section{GO-term analysis of beet miRNA targets}

The function of the target gene set was subjected to GO-term analysis, which enabled us to characterize the miRNA-gene regulatory network in terms of molecular functions, biological processes, and cellular components. Our study revealed 23 target genes that participate in 39 molecular functions; 22 of the genes are involved in biological processes and 8 of them are involved in cellular functions. GO-term enrichment analysis revealed that eight of the 12 newly identified beet miRNA families are involved in 17 biological processes, including actin cytoskeleton organization, ATP hydrolysis-coupled proton transport, chlorophyll biosynthesis, visible light detection, DNA replication, glycerophospholipid metabolism, lignin catabolism, nucleosome assembly, oxidation-reduction, photosynthesis, peptidyl-pyrromethane cofactor linkage, transcription regulation, DNA-templated sodium transport, tetrapyrrole biosynthesis, translational elongation, and transmembrane transport ( $\underline{\text { Table S1 }}$ ).

\section{CONCLUSIONS}

B. vulgaris has received significant attention throughout human history because of its nutritional and economic value. Beet cultivation is undergoing resurgence with increasing sugar and bioenergy production. However, the role of beet miRNAs and their target genes in various metabolic processes has remained elusive. In this context, comparative genome-based prediction and characterization of miRNAs provide an alternative to RNAimediated technology. For the first time, we identified 13 B. vulgaris miRNAs belonging to 12 families from 29,857 ESTs and 279,223 GSSs, using an in silico approach. These 12 miRNA families target 25 mRNAs. Most of the conserved miRNAs target transcription factors as well as genes involved in signal transduction, stress tolerance, and various metabolic processes, which are expected to provide miRNA-mediated gene regulation in this important plant. To understand the mutual relationship between miRNAs and their candidate target genes in beet, an examination of differential expression levels using quantitative polymerase chain reaction (qRT-PCR) and 5' RLM-RACE analyses is underway in our laboratory. The results from this study will shed more light on the regulation of beet miRNAs and their targets in the near future.

\section{ACKNOWLEDGMENTS}

Research supported by the National Natural Fund Project (\#31000831), National Modern Agriculture Industry Technology System Project (\#CARS-21010202), Natural Fund Project of Heilongjiang Province (\#C200914). We thank all the members of Sugar beet industry research group for their participation in the study.

\section{Supplementary material}

\section{REFERENCES}

Altschul SF, Gish W, Miller W, Myers EW, et al. (1990). Basic local alignment search tool. J. Mol. Biol. 215: 403-410. Barrera F (2012). High throughput sequencing reveals novel and abiotic stress-regulated microRNAs in the inflorescences of rice. BMC Plant Biology. 12: 132-143.

Bartel DP (2004). MicroRNAs, Genomics, Biogenesis, Mechanism, and Function. Cell 116: 281-297. 
Chen X (2004). A microRNA as a translational repressor of APETALA2 in Arabidopsis flower development. Science 303: 202-225.

Dalmay T (2006). Short RNAs in environmental adaptation. Proc Biol Sci. 273: 1579-1585.

Dezulian T, Palatnik JF, Huson D and Detlef Weigel (2005). Conservation and divergence of microRNA families in plants. Genome Biol. 6: 13-38.

Dezulian T, Remmert M, Palatnik JF, Detlef W, et al. (2006). Identification of plant microRNA homologs. Bioinformatics 22: 359-360.

Ding D, Zhang L, Wang H, Zhijie Liu, et al. (2009). Differential expression of miRNAs in response to salt stress in maize roots. Ann. Bot. 103: 29-38.

Gandikota M, Birkenbihl RP, Höhmann S, Cardon GH, et al. (2007). The miRNA156/157 recognition element in the 3'UTR of the Arabidopsis SBP box gene SPL3 prevents early flowering by translational inhibition in seedlings. The Plant. 49: 683-693.

Jones-Rhoades MW and Bartel DP (2004). Computational identification of plant microRNAs and their targets, including a stress-induced miRNA. Mol. Cell. 14: 787-799.

Jones-Rhoades MW, Bartel DP and Bartel B (2006). MicroRNAs and their regulatory roles in plants. Annu Rev Plant Biol. 57: 19-53.

Khraiwesh B, Zhu JK and Zhu JH (2012). Role of miRNAs and siRNAs in biotic and abiotic stress responses of plants. Biochim. Biophys. Acta 1819: 137-148.

Kulcheski, Luiz FV de Oliveira, Lorrayne G Molina, Maurício P Almerão, et al. (2011). Identification of novel soybean microRNAs involved in abiotic and biotic stresses. BMC Genomics. 12: 307-324.

Li HY, Dong YY, Yin HL, Wang N, et al. (2011). Characterization of the stress associated microRNAs in Glycine max by deep sequencing. BMC Plant Biology. 11: 170-182.

Lin F, Zhang Y and Jiang MY (2009). Alternative splicing and differential expression of two transcripts of nicotine adenine dinucleotide phosphate oxidase B gene from Zea mays. Plant Biol. 51: 287-298.

Llave C, Xie Z, Kasschau KD and Carrington JC (2002). Cleavage of Scarecrow-like mRNA targets directed by a class of Arabidopsis miRNA. Science. 297: 2053-2056.

Prabu GR and Mandal AKA (2010). Computational identification of miRNAs and their target genes from expressed sequence tags of tea (Camellia sinensis). Genomics Proteomics Bioinforma. 8: 113-121.

Sakaguchi J and Watanabe Y (2012). MiR165/166 and the development of land plants. Dev. Growth Differ. 54: 93-99.

Shukla L, Chinnusamy V and Sunkar R, (2008). The role of microRNAs and other endogenous small RNAs in plant stress responses. Biochim Biophys Acta. 1779: 743-748.

Song CN, Wang C, Zhang CQ, Korir NK, et al. (2010). Deep sequencing discovery of novel and conserved microRNAs in trifoliate orange (Citrus trifoliata). BMC Genomics. 11: 431-443.

Sunkar R, Chinnusamy V, Zhu J and Zhu JK (2007). Small RNAs as big players in plant abiotic stress responses and nutrient deprivation. Trends Plant Sci. 12: 301-309.

Unver T and Budak H (2009). Conserved microRNAs and their targets in model grass species Brachypodium distachyon. Planta 230: 659-669.

Wakeel A, Asif AR, Pitann B and Schubert S (2011). Proteome analysis of sugar beet (Beta vulgaris L) elucidates constitutive adaptation during the first phase of salt stress. J. Plant Physiology. 168: 519-526.

Yanhui C, Xiaoyuan Y, Kun H, Meihua L, et al. (2006). The MYB transcription factor superfamily of Arabidopsis, expression analysis and phylogenetic comparison with the rice MYB family. Plant Mol. Biol. 60: 107-124.

Zhang BH, Pan XP, Wang QL, Cobb GP, et al. (2005). Identification and characterization of new plant microRNAs using EST analysis. Cell Res. 15: 336-360.

Zhang BH, Pan X, Cannon CH, Cobb GP, et al. (2006a). Conservation and divergence of plant microRNA genes. Plant J. 46: 243-259.

Zhang BH, Pan X, Cobb GP and Anderson TA (2006b). Plant microRNA, a small regulatory molecule with big impact. Dev. Biol. 289: 3-16.

Zhang BH, Pan XP and Stellwag EJ (2008). Identification of soybean microRNAs and their targets. Planta 229: 161-182.

Zhang HW, Zhan JF, Ruidang Quan, Xiaowu Pan, et al. (2013). Ruidang Quan EAR motif mutation of rice OsERF3 alters the regulation of ethylene biosynthesis and drought tolerance. Planta. 237: 1443-1451 